\title{
The impact of proton-pump inhibitors on intraperitoneal sepsis: a word of caution for transgastric NOTES procedures
}

\author{
Sonia L. Ramamoorthy $\cdot$ Jeffrey K. Lee $\cdot$ Yoav Mintz $\cdot$ John Cullen \\ Michelle K. Savu · David W. Easter · Alana Chock - Ravi Mittal · \\ Santiago Horgan · Mark A. Talamini
}

Received: 6 December 2008 / Accepted: 1 May 2009/Published online: 24 June 2009

(C) The Author(s) 2009. This article is published with open access at Springerlink.com

\begin{abstract}
Background During transgastric natural orifice transluminal endoscopic surgery (NOTES), there is an iatrogenic perforation of the gastric wall with leakage of gastric contents into the peritoneal cavity. The aim of this study is to determine the effect of proton-pump inhibitors (PPI) and alterations of gastric $\mathrm{pH}$ on infection during transgastric surgery.

Methods Thirty 250-g male Sprague-Dawley rats were divided into a study group (SG, $n=15$ ) and a control group (CG, $n=15$ ). SG were given $5 \mathrm{mg} / \mathrm{kg}$ pantoprazole for 3 days before procedure and another dose $1 \mathrm{~h}$ before. CG received saline at similar time points. A mini-laparotomy with gastrotomy was performed. Aspiration of $2.0 \mathrm{cc}$ gastric contents was removed from the stomach and injected into the peritoneal cavity of both groups. Gastric $\mathrm{pH}$ and peritoneal $\mathrm{pH}$ levels were obtained. Gastric aspirate was sent for culture. White blood cell counts (WBC) were obtained on postoperative days 1,7 , and 14 , and C-reactive
\end{abstract}

Supported by Ethicon/NOSCAR 2007 research grant

Presented at the Society of American Gastrointestinal and Endoscopic Surgeons, Philadelphia, PA, 2008.

S. L. Ramamoorthy $(\bowtie) \cdot$ Y. Mintz $\cdot$ J. Cullen

M. K. Savu - D. W. Easter - S. Horgan - M. A. Talamini Center for the Future of Surgery- Department of Surgery, UC San Diego Medical Center, 3855 Health Sciences Drive, Suite 2073, La Jolla, CA 92093-0987, USA

e-mail: sramamoorthy@ucsd.edu

J. K. Lee $\cdot$ R. Mittal

Department of Medicine, San Diego Medical Center, University of California, San Diego, CA, USA

A. Chock

Northwest Weight Loss Surgery, 125 30th St. SE, Everett, WA 98208, USA protein (CRP) levels were obtained on postoperative day 1 . At day 14, a necropsy was performed and aerobic and anaerobic cultures of the peritoneal cavity were obtained. Results There were no deaths in either group. The average gastric $\mathrm{pH}$ in the $\mathrm{SG}$ was 5.13 versus $3.26(p=0.03)$ in the CG. The average peritoneal $\mathrm{pH}$ was similar in both groups. The WBC in the SG was 4.5 vs. 3.5 (1,000 cells/ $\mathrm{mm}$ ) in the CG. There was no elevation in CRP levels in either group. Bacterial cultures were positive in $3 / 15(20 \%)$ rats in the CG and in $9 / 15(60 \%)$ in the SG $(p=0.008)$. Intra-abdominal abscesses were found in $2 / 15(13 \%)$ rats in the CG and in 5/15 (33\%) in the SG $(p=0.08)$.

Conclusions Pretreatment with a PPI resulted in a higher rate of peritoneal bacterial contamination and abscess formation. The acidic environment of the stomach appears to be protective against infection when intraperitoneal contamination occurs as a result of gastrotomy.

Keywords Transgastric surgery $\cdot$ Natural orifice surgery · NOTES · Proton-pump inhibitors

Transgastric surgery is a form of natural orifice surgery that involves endoscopic gastrotomy followed by intraperitoneal surgery and gastric closure. The first reported cases of natural orifice transluminal endoscopic surgery (NOTES) were appendectomies performed by Reddy and Rao in India (personal communication with co-author, Talamini). The field of transgastric surgery is cautiously emerging as issues of safety and efficacy are investigated. A concern of transgastric surgery is spillage of gastric contents during transgastric passage. Much of the recent interest in "duallumen" and "hybrid" approaches to NOTES procedures stems from the concern over leakage and reliable closure. Historically, the leakage of gastric contents into the 
peritoneal cavity is thought to lead to peritonitis from both chemical and bacterial exposure. Much of these concerns arise from the surgical experience with perforated gastroduodenal ulcers. In most cases, patients are in extremis when presenting with perforated gastric disease and require emergent exploration and repair. Recent studies however point towards conservative management in a select population of patients who are believed to have the perforation "sealed". This management includes the use of antisecretory drugs such as proton-pump inhibitors (PPI), antibiotics directed at H. Pylori, and bowel rest [1]. PPIs are also prescribed for a variety of other reasons including reflux disease and chronic gastritis, post bariatric surgery, and are often used by patients on an intermittent basis for symptomatic relief from any number of upper gastrointestinal (GI) complaints [2]. In 2005, spending on PPIs exceeded US $\$ 12$ billion in the USA, making prescription PPIs the second largest therapeutic drug class in terms of sales [3]. The impact of PPIs on transgastric NOTES procedures is of particular interest as one of the anticipated benefits of natural orifice surgery is to minimize the physiologic impact of surgical intervention. The aim of this study therefore is to determine the effect of PPIs and alterations in gastric $\mathrm{pH}$ on infection during transgastric surgery.

\section{Materials and methods}

The following study was conducted after approval from and under supervision of the University of California, San Diego (UCSD) Institute for Animal Care and Use Committee (IACUC protocol \#S07035).

\section{Animals}

Thirty male Sprague-Dawley rats (250-275 g) were obtained from a US Department of Agriculture (USDA)approved vendor (Charles-Rivers Labs, Boston, MA). The rats were acclimatized to their environment for 3-5 days upon arrival, per IACUC protocol. During this period all animals were caged per UCSD animal care protocol with standard husbandry procedures, and given food and water ad libitum, overseen by UCSD Animal Care. The animals were kept in the institutional animal care facilities with automated $12 \mathrm{~h}$ of light-dark cycle maintained at $25^{\circ} \mathrm{C}$ room temperature.

\section{Protocol}

Animals were equally divided into a study group (SG, $n=$ 15) and a control group ( $\mathrm{CG}, n=15)$. The study group received subcutaneous pantoprazole (Wyeth Pharmaceuticals Inc., Philadelphia, PA) at a dose of $5 \mathrm{mg} / \mathrm{kg}$ injected subcutaneously under the neck fur every $8 \mathrm{~h}$ for 3 days and $1 \mathrm{~h}$ before the procedure. Standard PPI doses for rats vary in the literature between 5 to $20 \mathrm{mg} / \mathrm{kg}$ [4, 5]. The current dose was chosen to obtain noticeable change in gastric $\mathrm{pH}$ in this rat model. The control group was injected in similar fashion with sterile $0.9 \%$ normal saline at the same time points.

Preoperative care and anesthesia

The animals were weighed and given a study number. Three to four hours prior to procedure animals were fasted, placed in wire-based cages to prevent ingestion of bedding/stool/ food, and given access to water ad libitum. The animals were transferred to a surgical laboratory facility on the day of the procedure. Anesthesia was administered via a precision vaporizer. Induction was obtained with $4-5 \%$ inhaled isoflurane (IsoFlo; Abbot Laboratories, North Chicago, IL) and maintained with $2-3 \%$ isoflurane with $100 \%$ oxygen carrying gas. Depth of anesthesia was determined by spontaneous movement, twitching, increased respiratory rate, increased work of breathing, movement of extremities upon stimulation of plantar surface, and tail and toe pinch. After induction, the left upper quadrant was shaved using electric clippers, and sterilely prepped and draped. At the minilaparotomy incision site, $0.25 \%$ Marcaine $1 \mathrm{mg} / \mathrm{kg}$ (Astra-Zeneca, Wilmington, DE) was injected for pain control.

\section{Surgery}

After sterile draping of the animals, baseline serum complete blood counts were obtained using ventral tail artery or the lateral saphenous vein as access. Local anesthetic was then administered to the incision site in the left upper quadrant of the animal. A small $(<1 \mathrm{~cm})$ incision was made using sterile instruments (Fig. 1). After gaining access to the peritoneal cavity, baseline peritoneal $\mathrm{pH}$ levels were measured using an Accumet AB15 Basic benchtop pH meter (Fisher Scientific International Inc., Hampton, NH) and a MI-508 pH microelectrode (Microelectrodes Inc., Bedford, NH). This was done by placing the probe in a dependent portion of the peritoneal cavity posterior to the liver such that the tip of the catheter is constantly bathed in the small amount of peritoneal fluid present there. This technique has been previously described by Hanly et al. [6]. A reference electrode (Microelectrodes Inc.) was inserted into the right ear. The system was calibrated before each animal by immersing the tips of the $\mathrm{pH}$ and reference electrodes in sterile commercially prepared buffer solutions (Fisher Scientific, Fair Lawn, NJ) of pH 7.0 and $\mathrm{pH}$ 4.0. Once the baseline peritoneal $\mathrm{pH}$ values for each animal were obtained, the stomach was exposed and baseline gastric $\mathrm{pH}$ values (an average of three readings) were recorded. Needle aspiration (16G) of gastric contents 


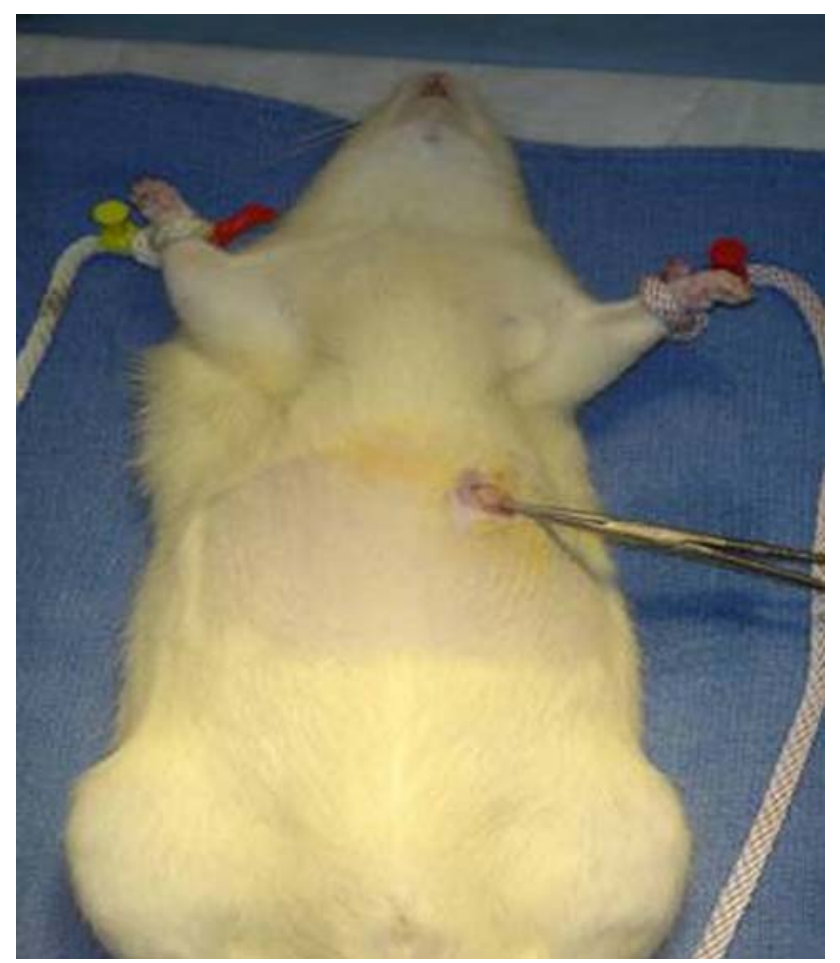

Fig. 1 Animal surgery: minilaparotomy in left upper quadrant

was performed into a syringe containing $2 \mathrm{cc} 0.9 \%$ normal saline. As a control, a sample of the gastric/saline mix was rechecked for changes in $\mathrm{pH}$; none was noted. This material was then injected into the peritoneal cavity of both the study and control groups to mimic gastric spillage during transgastric natural orifice surgery. The needle insertion site in the stomach was closed using an absorbable stitch. The fascia and muscle were closed using an absorbable suture and the skin reapproximated using Indermil ${ }^{\circledR}$ tissue adhesive. Animals were given buprenorphine $0.05 \mathrm{mg} / \mathrm{kg}$ subcutaneously prior to recovery (Reckitt Benckiser Pharmaceuticals, Richmond, VA.) for pain control. Total surgery time ranged from 10 to $15 \mathrm{~min}$. Animals were recovered in postprocedure cage, with a heating source and monitoring. Animals were monitored until they were able to maintain sternal recumbency and were normothermic.

\section{Recovery and postoperative observation}

The animals were returned to feed ad libitum. White cell count was drawn on postoperative days (POD) 1, 7, and 14 . Daily weights, eating habits, clinical signs of sepsis (piloerection, abdominal tenderness, and lethargy), and bowel movements were assessed on each rat. On POD 14, the rats were sacrificed and subject to gross examination. Abscess was defined as the presence of purulent material within the peritoneal cavity at the time of necropsy.
Cultures

Aerobic and anaerobic cultures were sent from the gastric aspirate and again at the time of necropsy. Cultures were obtained by swab and delivered to the UCSD microbiology laboratory within $1 \mathrm{~h}$ of procedure. The swabs were applied to aerobic and anaerobic culture media using standard CLIA-approved procedure. The swabs were placed on appropriate agar plates and three streaks made per plate. Growth on only the first streak, which is considered "scant" or $(1+)$ culture, was quantified as $<10-20$ colony forming units (CFUs). Growth on streaks $2-3$ is considered moderate $(2+)$ to heavy growth $(3-4+)$. The cultures were then speciated by UCSD microbiology laboratory based on microscopic evaluation.

\section{Statistical analysis}

Descriptive statistics were used throughout. Nonparametric Student's $t$-test was used to analyze differences between the control and study group. Statistical significance was reported as those $p$ values of less than 0.05 .

\section{Results}

There were no deaths or leaks in either group. The average intragastric $\mathrm{pH}$ in the study group was 5.13 versus $3.26(p=$ 0.03 ) in the control group. The average intraperitoneal $\mathrm{pH}$ was similar in both groups ( 7.09 versus $7.19, p=0.34$ ). The white blood cell count in the study group increased by an average of 4.5 versus $3.5(1,000$ cells $/ \mathrm{mm})$ in the control group. There was no elevation in CRP levels in either group. Bacterial cultures were positive in 3/15 (20\%) rats in the control group and in $9 / 15(60 \%)$ in the group treated with PPI $(p=0.008)$. Intra-abdominal abscesses were found in 2/15 (13\%) rats in the control group and in 5/15 (33\%) in the group treated with PPI $(p=0.08)$. The most common isolate in the rat cultures were E. coli, Proteus mirabilis, and Staphylococcus xylosus. There was a higher rate of $E$. coli infection $(>3+)$ in the PPI treated group 8/9 (88\%) compared with the control group 1/3 (33\%) (Table 1).

\section{Discussion}

In this rodent model, we studied the effect of PPIs on intraabdominal sepsis after gastric spillage. From our results, we saw a higher rate of bacterial contamination and abscess formation in the study group. While our data for abscess was not statistically significant, there appeared to be a trend towards higher rate of infection. Of the cultures that were positive, there was a higher rate of Gram-negative (enteric) 
Table 1 Results of positive gastric cultures

\begin{tabular}{llll}
\hline Bacteria & Control group & Study group & $p$ value \\
\hline Positive cultures & $3 / 15(20 \%)$ & $9 / 15(60 \%)$ & 0.008 \\
Abscess & $2 / 15(13 \%)$ & $5 / 15(33 \%)$ & 0.08 \\
AEROBES & & & \\
S. Xylosus & $1+$ & $3+$ & \\
S. Sciuri & $1+$ & $2+$ & \\
S. Aureus & $1+$ & $2+$ & \\
Strep. sp & $1+$ & $2+$ & \\
Staph sp & $1+$ & $1+$ & \\
ANAEROBES & & & \\
E. Coli & $1+$ & $4+$ & \\
Proteus Mirabilis & $4+$ & $4+$ & \\
Proteus sp. & $2+$ & $3+$ & \\
Enterobacter sp. & $1+$ & $2+$ & \\
Lactobacillus & $4+$ & $1+$ & \\
\hline
\end{tabular}

Results represent average growth from positive cultures

infection in the PPI-treated group versus the control group. This data supports the conclusion of several studies on rodents and humans that found an association between acid suppression and bacterial overgrowth/infection [6-9]. In a landmark study by Franklin et al., it was found that the previously held notion of a "sterile" foregut was challenged by the finding of polymicrobial gastric flora. This study was the first to show an increase in colony-forming units (CFUs) and an increase in enteric pathogens as the gastric $\mathrm{pH}$ increases [10]. Our study observed similar findings with loss of comensural organisms such as Lactobacillus, and increased growth of enterics such as E. coli and Proteus species. More recently, the chronic use of PPI has been associated with community-acquired Clostridium difficile colitis [11] and a meta-analysis found an association with acid suppression and enteric infection [12]. Similarly, in two retrospective human studies, the crude odds ratio for development of spontaneous bacterial peritonitis among PPI users versus nonusers ranged from 1.22 to $7.0[8,13]$. A human study by Narula et al. found that transgastric instrumentation contaminates the peritoneal cavity and those patients on PPIs have a higher bacterial load compared with nonusers [7]. In this small series of 50 patients, there was no observable adverse clinical outcome as a result of a higher bacterial load; however these patients were all treated with preoperative antibiotics. Our study demonstrates similar clinical findings to those of Narula, although with the added benefit of the animal model, we were able to demonstrate a higher rate of peritoneal infection with a trend towards significance $(p=0.08)$ in the study group. Despite these findings, there was no observable difference between the groups with regards to subjective or objective criteria such as behavior, activity level, absolute neutrophil count or C-reactive proteins levels. Our rat model raises the question as to whether the alterations in gastric $\mathrm{pH}$ as a result of PPIs and subsequent changes in gut flora are of clinical significance necessitating changes in clinical practice.

The limitations of our study was that we worked with a set "volume" of gastric aspirate that was chosen as a percentage of total body weight of the rat model, i.e., $2 \mathrm{cc} /$ $250 \mathrm{~g}$ rat $=320 \mathrm{cc} / 60 \mathrm{~kg}$ human. It is difficult to assess whether an increase in bacterial load or "volume" would have led to a different outcome, or whether one can extrapolate human outcomes from a rodent model. In addition, our experiment design could have more closely approximated the clinical scenario of NOTES by using open or closed gastrotomy with peritoneal contamination instead of directly injecting gastric contents into the peritoneum, which may have caused an increase rate of abscess formation.

The results of this study are suggestive of an increased risk of intraperitoneal infection with proton-pump inhibitors, but it remains unclear if simple measures such as appropriate antibiotic coverage, aggressive sterile technique, and better clearance of secretions will reduce this risk. More investigations are warranted to address this issue before alterations in clinical practice can be recommended.

Open Access This article is distributed under the terms of the Creative Commons Attribution Noncommercial License which permits any noncommercial use, distribution, and reproduction in any medium, provided the original author(s) and source are credited.

\section{References}

1. Bucher P, Oulhaci W, Morel P, Ris F, Huber O (2007) Results of conservative treatment for perforated gastroduodenal ulcer in patients not eligible for surgical repair. Swiss Med Weekly 1137:337-340

2. Vanderhoff B, Tahboub RM (2002) Proton pump inhibitors: an update. Am Family Phys 66(2):273-280

3. IMS Health. Leading 20 therapeutic classes by U.S. sales, 2005. http://www.imshealth.com. Accessed Dec 2007

4. Bonin EA, Campos AC, Coelho JC, Matias JE, Malafaia O, Jonasson TH (2005) Effect of pantoprazole administered subcutaneously on the healing of sutured gastric incisions in rats. Eur Surg Res 37(4):250-256

5. Ferron GM, McKeand W, Mayer PR (2001) Pharmacodynamic modeling of pantoprazole's irreversible effect on gastric acid secretion in humans and rats. J Clin Pharmacol 41(2):149-156

6. Hanly EJ, Aurora AR, Fuentes JM, Shih SP, Marohn MR, De Maio A et al (2005) Abdominal insufflation with $\mathrm{CO}_{2}$ causes peritoneal acidosis independent of systemic $\mathrm{pH}$. J Gastrointest Surg 9(9):1245-1251

7. Narula VK, Hazey JW, Renton DB, Reavis KM, Paul CM, Hinshaw KE et al (2008) Transgastric instrumentation and bacterial contamination of the peritoneal cavity. Surg Endosc 22:605-611 
8. Campbell MS, Obstein K, Reddy KR, Yang YX (2008) Association between proton pump inhibitor use and bacterial peritonitis. Dig Dis Sci 53:394-398

9. Theisen J, Nehra D, Citron D, Johansson J, Hagen JA, Crookes PF et al (2000) Suppression of gastric acid secretion in patients with gastroesophageal reflux disease results in gastric bacterial overgrowth and deconjugation of bile acids. J Gastrointest Surg 4(1):50-54

10. Franklin MA, Skoryna SC (1966) Studies on natural gastric flora. I. Bacterial flora of fasting human subjects. Can Med Assoc J 95(26):1349-1355
11. Dial S, Delaney JA, Barkun AN, Suissa S (2005) Use of gastric acid-suppressive agents and the risk of community-acquired clostridium difficile-associated disease. JAMA 294:2989-2995

12. Leonard J, Marshall JK, Moayyei P (2007) Systematic review of the risk of enteric infection in patients taking acid suppression. Am J Gastroenterol 24:2047-2056

13. Bauer TM, Steinbruckerner B, Brinkmann FE, Ditzen AK, Aponte JJ, Pelz K et al (2001) Small intestinal bacterial overgrowth in patients with cirrhosis: prevalence and relation with spontaneous bacterial peritonitis. Am J Gastroenterol 96:29622967 